\title{
Progrès génétique en colza et perspectives
}

\author{
Xavier PINOCHET ${ }^{1}$ \\ Michel RENARD ${ }^{2}$ \\ ${ }^{1}$ CETIOM, \\ Centre de Grignon, \\ Avenue Lucien-Brétignières, \\ 78850 Thiverval-Grignon, \\ France \\ <pinochet@cetiom.fr> \\ 2 UMR 1349, \\ IGEPP INRA Agro Campus Ouest \\ Université Rennes 1, \\ BP 35 327, \\ 35653 Le Rheu Cedex
}

Depuis la réintroduction de cultures oléagineuses en France après la seconde guerre mondiale, l'orientation principale prise par la génétique et l'amélioration du colza a été la productivité en grain et en huile pour répondre à des marchés déficitaires aussi bien en huile, qu'en protéines pour l'alimentation animale. Cette situation a prévalu jusqu'à présent. Après quelques rappels historiques sur le développement de la culture du colza et en parallèle l'évolution des techniques d'amélioration des plantes, nous dresserons un bilan du progrès génétique enregistré. Depuis quelques années, les objectifs de sélection se diversifient sous les influences conjuguées de la diversification des marchés à satisfaire, de l'évolution des réglementations et des souhaits de la société. La génétique a en particulier un rôle primordial à assurer dans le cadre de la révision générale des stratégies de protection des cultures engagée via ECOPHYTO 2018, avec I'objectif particulièrement ambitieux de réduire de $50 \%$ les applications de produits phytosanitaires. Le secteur de l'amélioration des plantes essaie de répondre à ces attentes en diversifiant les caractères pris en compte, en maintenant ou

\begin{abstract}
Rapeseed genetic progress and outlook for developments
Since the end of the fifties the main aim of genetics and plant breeding for WOSR was grain and oil productivity to provide unsatisfied markets for oil as well as for animal feeding. In the following periods others objectives were added in the field of product quality and resistance to diseases. After an historical perspective of the crop development and the parallel evolution of breeding technics, we will set a balance sheet of the genetic progress reached. For several years now, breeding objectives are more diverse coming from market diversification, regulations behavior, or societal wishes. Genetics and plant breeding have specifically an important role to play in the field of new plant protection strategies. Today strategies will be carried out to satisfy such aims through wider genetic diversity exploitation, and genotyping and phenotyping methods improvements.
\end{abstract}

Key words: oilseed rape, genetic improvement, productivity

en élargissant la diversité génétique travaillée, en modernisant ses méthodes aussi bien au niveau des génotypages que des phénotypages. Cette politique redonne également un intérêt à la relance des études des interactions Génotypes $\times$ milieux $\times$ conduites pour optimiser productivité et impacts dans un grand nombre de situations.

\section{Un peu d'histoire}

En France et en Europe la culture du colza est ancienne. Cette plante dont on soupçonne l'apparition il y a environ 1000 ans, était utilisée principalement pour produire de I'huile destinée à I'éclairage. Au milieu du XIX ${ }^{\mathrm{e}}$ siècle, les surfaces consacrées au colza sont de l'ordre de 200000 ha. Les principales zones de culture sont les bordures maritimes de la Manche, du Pas de Calais au Cotentin, la Bretagne et les Pays de Loire. Le colza est présent ailleurs mais de façon diffuse à petite échelle. Néanmoins, la production a fortement diminué pour quasiment disparaître à la fin du $\mathrm{XIX}^{\mathrm{e}}$ siècle. En parallèle des conquêtes coloniales en Afrique et en Asie du Sud-Est, les productions $d$ 'huile nécessaires au pays se sont progressivement déplacées dans les colonies, avec les productions d'Arachide et de palme. Ainsi les productions oléagineuses ont quasiment disparu des assolements français de la fin du second empire à la fin de la seconde guerre mondiale. Ce n'est qu'à partir des années cinquante, que le colza a été progressivement réintroduit. Les zones dites aujourd'hui traditionnelles de culture du colza comme la Bourgogne ou le Berry, sont en fait des zones de culture récente à l'échelle historique. La progression des surfaces a été régulière, de 100000 ha en 1960 à 1500000 ha à partir de 2006 (figure 1). Les quelques à-coups sont liés à la crise de l'acide érucique dans les années 70 puis aux réformes de la Politique agricole commune de 1992, et de 1999, qui ont modifié les conditions de compétitivité du colza par rapport aux autres cultures.

Les travaux de sélection du colza ont commencé en France à I'INRA au milieu des années cinquante. La première étape importante a été la mise en place du système $d$ 'inscription des variétés et la création du CTPS au début des années 60. La réglementation impose, pour des raisons de protection et de reconnais-

Pour citer cet article : Pinochet X, Renard M. Progrès génétique en colza et perspectives. OCL $2012 ; 19(3)$ : 147-154. doi : 10.1684/ ocl.2012.0456 
sance du travail des sélectionneurs, le principe de lignées pures homozygotes. Ceci permet d'apprécier plus facilement le progrès apporté par chaque génotype nouveau et d'offrir une garantie à l'agriculteur sur l'identité du matériel qu'il achète. Cette garantie est donnée au travers d'épreuves DHS (Distinction, Homogénéité, Stabilité en autofécondation) et VAT (valeur agronomique et technologique) obtenues à partir d'un réseau d'essais représentatifs des conditions d'utilisation. Ces dispositions créent les conditions d'un accroissement du progrès génétique sur la base de variétés lignées pures homozygotes alors que les colzas cultivés précédemment étaient des variétés populations hétérogènes, par définition sans aucune garantie de stabilité pour l'agriculteur.

Pendant une trentaine d'années de 1960 à 1990, I'amélioration génétique du colza a été réalisée en France par une association unique entre I'INRA et les établissements Ringot. Les premiers objectifs de sélection ont été l'accroissement de la productivité et l'amélioration de la résistance au Phoma. Objectif atteint en particulier par l'inscription en 1970 de la variété Ramsès.

$\mathrm{Au}$ cours de son histoire la sélection du colza a connu plusieurs étapes délicates.

La première a été celle de l'acide érucique. À la fin des années 60 , des médecins et nutritionnistes ont reproché à I'huile de colza sa composition en acide gras, principalement constituée pour 45 à $50 \%$, d'acide érucique. En quelques années, les sélectionneurs ont créé des variétés ne contenant plus d'acide érucique, mais environ 60 à $70 \%$ d'acide oléique. Ce résultat a été obtenu par rétrocroisements en utilisant comme géniteur une lignée de printemps canadienne sans acide érucique et la variété "Major » comme parent récurrent. Le déterminisme relativement simple du caractère, la possibilité de sélectionner ce caractère sur demigraines et de réaliser deux cycles de sélection par an en conditions contrôlées ont permis d'aboutir en un temps record à la variété "Primor " inscrite en 1973, laquelle a ainsi été la première variété 0 du catalogue français, bientôt suivi de la variété "Jet Neuf " qui a dominé les assolements européens pendant une dizaine d'années à partir de 1978, puis «Bienvenue » en 1983.
La seconde fut la nécessaire reconversion du matériel en variétés à faible teneur des graines en glucosinolates. Cette reconversion a été obtenue progressivement avec d'abord la variété "Darmor" (inscription en 1983) à teneur intermédiaire, avant "Samourai " (inscription en 1989) dont les teneurs en glucosinolates des graines sont proches de celles des variétés actuellement cultivées.

À la fin des années 70 et au tournant des années 80 plusieurs évolutions méthodologiques ont révolutionné I'amélioration génétique du colza. II s'agit de la mutagénèse chimique, de I'Haplodiploïsation et enfin de la fusion de protoplastes.

La mutagénèse chimique à l'EMS (Ethyl Méthane Sulfonate) réalisée par I'INRA à partir du milieu des années 70 avait pour objectif de générer de la variabilité génétique dans une espèce réputée reposer sur une base de ressources génétiques relativement réduite. Ceci a permis d'identifier au fil du temps des lignées à caractères agronomiques originaux pouvant avoir un intérêt appliqué significatif. On peut citer quelques exemples, comme des mutants sur les désaturases qui ont permis l'accès à une série de profils d'acides gras originaux, le mutant nain (protection par brevet de l'utilisation du gène de nanisme Bzh) qui a abouti à l'émergence des hybrides demi-nains (Lutin en 1999), ou un mutant cléistogame dont la fleur reste en forme de tulipe, limitant ainsi les échanges de pollen entre plantes et entre parcelles et favorisant ainsi l'autogamie. L'impact de ce caractère a été étudié de près pour limiter les disséminations de pollen dans I'hypothèse de recours en colza aux technologies OGM, ou pour permettre la coexistence dans un même environnement de variétés ayant des profils d'acides gras différents.

L'haplodiploïdisation a été développée pour le colza à partir du début des années 80. Elle permet par culture de microspores puis doublement chromosomique à la colchicine d'obtenir directement à partir d'un croisement des lignées fixées parfaitement homozygotes. Cette technique a eu un impact très important sur le monde de la sélection du colza. Il devenait en effet possible, à partir de croisements utilisant en particulier des variétés commerciales élites, de générer un grand nombre de lignées fixées parmi lesquelles ont été sélectionnées de nouvelles variétés. Le travail de sélection est alors centré sur un travail de phénotypage, avec des délais considérablement raccourcis entre le croisement initial et le dépôt à I'inscription. Ainsi, il a été envisageable de monter des programmes de sélection du colza à des coûts relativement réduits et surtout avec des perspectives de création variétale rapide. C'est à partir de la disponibilité de cette technique d'haplodiploïdisation, en plus de la sélection généalogique, que sont apparus des programmes concurrents de celui de l'opérateur historique INRARingot (puis Serasem). La plupart des sociétés semencières françaises et européennes ont alors mis en place des programmes de sélection pour le territoire français. Nous sommes passés d'un unique programme historique à une quinzaine de programmes issus de grandes sociétés, mais aussi beaucoup de sociétés plus petites dont certaines ont créé des variétés leaders de marché. Ceci s'est traduit à partir du début des années 90 par une explosion du nombre de variétés inscrites au catalogue français et l'accès pour le producteur à une plus grande diversité génétique.

Une troisième technique issue de la biologie cellulaire a eu également une grande importance pour les progrès de I'amélioration du colza, c'est la fusion de protoplaste. Elle a permis d'obtenir la fusion d'une cellule de colza conventionnel et d'une cellule de colza déficiente en chlorophylle portant le génome mitochondrial d'un radis conférant une stérilité male cytoplasmique. Ce résultat a été obtenu et publié dans le cadre d'une collaboration exemplaire entre chercheurs de I'INRA de Versailles et de Rennes en 1983 (Pelletier et al., 1983). Ce matériel s'est ensuite avéré comme une excellente stérilité male, particulièrement stable, et a servi de base au développement des composites hybrides lignées au milieu des années 90 ("Synergy " inscrite en 1994), puis des hybrides restaurés à partir de la fin des années 90 lorsque la restauration a pu être introduite à partir d'un radis dit restaurateur. Le système Ogu-INRA issu de ces travaux, et protégé par brevet, est aujourd'hui le système leader en matière de fabrication des hybrides.

Une technique de biotechnologie a marqué ces dernières années par les débats et controverses qu'elle a créés. II $s^{\prime}$ agit de la transgénèse. Elle consiste à 
introduire et à faire $s^{\prime}$ exprimer dans un organisme vivant une séquence d'ADN originaire d'une autre espèce pour conférer un caractère particulier. L'élément novateur et controversé est que cette technique en permettant de s'affranchir de la reproduction sexuée permet de franchir la barrière des espèces. Cette technique a été élaborée à partir de la découverte au début des années 70 de la faculté de bactéries du sol du genre Agrobacterium d'intégrer au génome de leur plante hôte une séquence d'ADN portée par un plasmide. La première plante OGM a été obtenue à Gant en Belgique sur Tabac en 1983. Très vite ensuite les chercheurs ont été capables de transformer d'autres espèces parmi lesquelles le colza à partir de 1988.

Les généticiens du colza avaient fondé de grands espoirs dans cette technique avec de très nombreuses applications (tableau 1) aussi bien en protection des cultures, que pour des aspects de qualité des graines. Le moratoire de février 1997 et les réticences de la société, n'ont pas permis le développement de cette technologie en Europe, alors que les équipes européennes étaient pourtant pionnières.

L'amélioration des plantes a poursuivi son travail avec des techniques et des approches plus classiques. C'est à partir du début des années 90 que vont se produire une série d'évolutions majeures au niveau des techniques de biologie moléculaire. Les cartes génétiques sont d'abord construites avec des marqueurs RAPD puis RFLP. Ceux-ci sont ensuite remplacés par des marqueurs SSR (microsatellites). Les cartes génétiques qui en sont issues portent sur quelques centaines de marqueurs qui laissent des intervalles entre eux importants, rendant les QTL identifiés souvent larges et donc peu précis. L'avènement des premiers séquençages complets au début des années 2000, puis la révolution des techniques de séquençage au milieu des années 2000 va permettre

Tableau 1. Liste des applications de la transgénèse chez le colza, aujourd'hui abandonnées pour la plupart faute d'être acceptées par la société ou en raison du coût prohibitif de l'homologation. D'autres applications non listées sont également prêtes à l'état de gènes clonés et validés par transformation.

\begin{tabular}{|c|c|c|c|}
\hline Caractère introduit & Effet attendu & Stade de développement & Références \\
\hline Résistance Glyphosate & Protection contre adventices & $\begin{array}{l}\text { Essais au champ, développement } \\
\text { commercial au Canada }\end{array}$ & $\begin{array}{l}\text { Shah et al. US patent } 1990 \text {; } \\
\text { Simard et al., } 2002\end{array}$ \\
\hline Résistance Glufosinate & Protection contre adventices & $\begin{array}{l}\text { Essais au champ, développement } \\
\text { commercial au Canada }\end{array}$ & Dröge et al., 1992 \\
\hline Résistance Glufosinate + gène Bar & $\begin{array}{l}\text { Protection contre adventices } \\
\text { et productivité (hybride) }\end{array}$ & $\begin{array}{l}\text { Essais au champ, développement } \\
\text { commercial au Canada }\end{array}$ & Agence Canadienne 1995 \\
\hline Resistance Oxynils & Protection contre adventices & Essais au champ & Freyssinet et al., 1996 \\
\hline endochitinase & $\begin{array}{l}\text { Protection sclerotinia, alternaria, } \\
\text { Phoma et cylindrosporiose }\end{array}$ & Essais au champ & $\begin{array}{l}\text { Grezes-Besset et al., 1995, } \\
\text { Grison et al., } 1996\end{array}$ \\
\hline Oxalate oxydase de l'orge & Protection sclérotinia & Essais en serre & Freyssinet et al., 1995 \\
\hline Oxalate oxydase de blé & Protection sclérotinia & & $\begin{array}{l}\text { Thompson et al., 1995, } \\
\text { Dong et al., } 2008\end{array}$ \\
\hline $\begin{array}{l}\text { Surexpression synthèse } \\
\text { de lignine }\end{array}$ & Protection sclérotinia & & Yang et al., 2007 \\
\hline $\begin{array}{l}\text { PR5 thaumatin-like protein } \\
\text { HV-TLP8 de l'orge }\end{array}$ & Protection Hernie & $\begin{array}{l}\text { Essais en serres. } 3 \text { générations } \\
\text { successives }\end{array}$ & Reiss et al., 2009 \\
\hline PR du pois & $\begin{array}{l}\text { Protection contre Phoma, } \\
\text { Rhizoctonia et Sclérotinia }\end{array}$ & & Wang et Fristensky 2001 \\
\hline Inhibiteurs de protéase & Protection insectes et limacees & & Jouanin et al., 1998 \\
\hline Toxine Bt & $\begin{array}{l}\text { Protection contre mouche } \\
\text { du chou et lepidoptères }\end{array}$ & & Stewart et al., 1996 \\
\hline Lectine de pois & Protection contre méligèthes & & Ahman et al., 2009 \\
\hline $\begin{array}{l}\text { Hte teneur en ac } \\
\text { Erucique }(>70 \%)\end{array}$ & qualité & Essais en serre & Nath et al., 2009 \\
\hline $\begin{array}{l}\text { Hte teneur en acide } \\
\text { laurique }(C 12-0>30 \%\end{array}$ & qualité & $\begin{array}{l}\text { Essais au champ, développement } \\
\text { commercial aux USA }\end{array}$ & Voelker et al., 1992 \\
\hline $\begin{array}{l}\text { Colza HOLLI (C18-1 > } 75 \% \text {; } \\
\text { C18-3<3\%) }\end{array}$ & Qualité & & Cartea et al., 1998 \\
\hline $\begin{array}{l}\text { Hte teneur en acide } \\
\text { myristique (C14-0) }\end{array}$ & Qualité & Essais au champ & Rudloff et Wehling, 1997 \\
\hline Alanine Amino Transférase & Nutrition azotée & $\begin{array}{l}\text { Essais au champ, dév. commercial } \\
\text { aux USA et Canada }\end{array}$ & Good et al., 2007 \\
\hline Reduction egrénage & productivité & Essais en serres et au champ & $\begin{array}{l}\text { Ostergaard et al., 2006, } \\
\text { Hua et al., } 2009\end{array}$ \\
\hline
\end{tabular}


I'émergence des marqueurs SNP. Les cartes génétiques vont pouvoir ainsi être très fortement densifiés avec au moins 100 fois plus de marqueurs permettant ainsi de gagner en précision surl'identification des zones d'intérêt et facilitant le travail visant à identifier les fonctions physiologiques et les allèles favorables correspondants, pour définir des marqueurs fiables et utilisables en sélection. Pour ce travail d'identification du lien entre une zone du génome et sa fonction physiologique, les résultats acquis par les physiologistes moléculaires sur la plante modèle Arabidopsis constituent des ressources utiles pouvant faciliter le travail, malgré quelques obstacles comme la présence de plusieurs génomes ( $A$ et $C$ du colza), ou la duplication de certains gènes présents en plusieurs copies dont les expressions peuvent se compléter. Néanmoins, la pleine valorisation de ces progrès nécessite en parallèle un accroissement des capacités de phénotypage et le développement des méthodologies de sélection génomique.

\section{Bilan du progrès génétique}

Durant les dernières décennies, les efforts de la génétique ont principalement porté sur les gains de productivité et les moyens d'assurer la régularité des niveaux atteints, en particulier en travaillant la résistance aux maladies. Le troisième objectif majeur a été la qualité, avec en premier lieu l'augmentation de la teneur en huile, mais aussi les modifications de profils d'acides gras ou la teneur en glucosinolates des graines.

\section{Productivité}

Ce critère est intégrateur et constitue une résultante de différents efforts réalisés non seulement pour le rendement mais aussi sur les systèmes d'hybridation, sur des comportements en situation de stress divers, ou sur la déhiscence des siliques. Le graphique de la figure 2 retrace l'évolution des rendements moyens obtenus dans les essais CTPS. La progression moyenne enregistrée sur 50 ans est de l'ordre de 0,75 quintal/ha/an soit plus de $2 \%$ par an. Cet ensemble de résultats semble le plus approprié pour faire ce type d'évaluation, dans la mesure où les sites et les conditions de réalisation sont relativement stables dans le temps, même si il y a des évolutions comme par exemple la réduction des niveaux de fertilisation azotée.

La progression n'est pas linéaire. On voit en particulier les diminutions temporaires liées aux reconversions successives, zéro érucique dans les années 70 puis double zéro dans les années 80 .

Ces résultats cumulent l'ensemble des séries expérimentales et ne permettent donc pas la mise en évidence de l'impact positif de l'arrivée des hybrides, ni au contraire l'impact négatif de matériels comme les premières générations d'hybrides demi-nains ou comme les premières variétés à faible teneur en acide linolénique.

Dans une étude conduite par le GEVES en 2004 A. Luciani a distingué lignées et associations variétales. Elle montre un écart d'environ 2 quintaux par hectare en faveur des associations variétales. La progression enregistrée sur la période 1988-2000 est estimée à 1,4\% par an soit 0,54 quintal/ha/an. Depuis, au fil des campagnes d'évaluation variétale, les matériels hybrides à fertilité restaurée s'imposent. Les lignées sont devenues quasi absentes du CTPS, ainsi que les associations variétales, et commercialement les hybrides occupent $75 \%$ des surfaces en 2012 en France.

Si on regarde les évolutions au niveau des rendements moyens nationaux obtenus sur une longue période (figure 3), le gain de productivité observé est de l'ordre de 0.4 quintal/ha/an, avec des fluctuations plus importantes liées aux évolutions de surface et à la nature des milieux utilisés, à l'évolution et à la diversité des pratiques. On retrouve néanmoins l'impact des deux reconversions, érucique et basse teneur en glucosinolates, ainsi que des impacts d'années à fortes épidémies comme 1993 et 1994 avec un impact Phoma, 2000 et 2001 avec de fortes épidémies de pHoma et le contournement de la résistance RIm1, ou 2006 et 2007 avec beaucoup de Sclérotinia mal contrôlé.

\section{Résistance aux maladies}

La plupart des maladies du colza, au moins les principales, font l'objet de travaux de génétique et d'amélioration des plantes. Les progrès génétiques sont particulièrement importants pour le Phoma (maladie due au champignon Leptosphaeria maculans). Le Phoma est à I'heure actuelle la maladie la plus importante du colza aussi bien aux échelles française, européenne que mondiale. Les premiers travaux d'identification de sources de résistance datent des années 60 avec I'association de I'INRA et du sélectionneur privé Ringot qui aboutit en 1970 à l'inscription de la variété "Ramsès", première variété tolérante. Ensuite la variété " Jet Neuf » (inscription en 1977) apporte un excellent niveau de tolérance. Cette variété va régner pendant 10 ans sur l'ensemble du marché européen et va faire oublier la question du Phoma pendant les années 80 . Ce n'est qu'après la reconversion en variétés " 00 " à la fois sans acide érucique et à basses teneurs en glucosinolates, que la question du phoma fait sa réapparition, en particulier lors des deux campagnes successives de 1992-93 puis 1993-94. À partir du milieu des années 90, I'arrivée des variétés portant la résistance spécifique RIm1 vont permettre de contrôler la maladie. En parallèle les efforts de sélection vont être poursuivis sur les facteurs de résistance quantitative. Dans les années 2000, le niveau moyen de résistance des variétés commercialisées a considérablement progressé comme le montre la figure 4. Plus des deux tiers des variétés commercialisées sont aujourd'hui de type très peu sensible (TPS). Parmi les nouveaux hybrides, quelques-uns portent une nouvelle résistance spécifique efficace RIm7.

La cylindrosporiose se manifeste régulièrement en particulier lors d'hivers humides, et fait l'objet alors d'applications fongicides. Néanmoins, les nouvelles variétés sont systématiquement évaluées au CTPS pour la résistance à cette maladie. Environ $40 \%$ des variétés commercialisées sont très peu sensibles.

Les progrès génétiques sont également sensibles pour la résistance à la Hernie des crucifères, avec I'hybride Mendel qui porte une résistance spécifique, malheureusement contournée dans certaines situations. Les efforts des sélectionneurs aussi bien sur Choux que sur Colza devraient permettre l'arrivée prochaine sur le marché de variétés présentant de bons niveaux de résistance quantitative.

Les autres principales maladies du colza que sont le Sclérotinia ou le Verticillium font l'objet également de travaux de génétique significatifs qui devraient permettre l'arrivée de variétés plus résistantes dans un avenir assez proche. Il faut mentionner que dans le cas du 
Sclérotinia plusieurs stratégies OGM étaient sur le point de déboucher lorsque l'exploitation en production de ce type de technologie a été stoppée par les pouvoirs publics (tableau 1).

\section{Qualité}

L'histoire de la sélection du colza a été marquée par les deux reconversions que constituent au début des années 70 l'arrivée des variétés sans acide erucique puis au milieu des années 80 la mise sur le marché de variétés à la fois sans acide érucique et à basse teneur des graines en glucosinolates. Cette norme de qualité dite " 00 » est celle toujours en vigueur aujourd'hui (figures 2-5).

Sur le marché de commodité les variétés 00 ont été améliorées sur la teneur en huile. Néanmoins dans l'étude de Luciani (2004) les progrès n'apparaissent pas très significatifs malgré le système de bonus-malus mis en place au niveau du CTPS à titre d'incitation. Les progrès sont plus significatifs sur la teneur en glucosinolates des graines (GLS) comme l'illustre la figure 5. L'abaissement de ce niveau de GLS a été contrarié par les interférences avec le système de restauration Ogu INRA. Les premières générations d'hybrides restaurés avaient des teneurs un peu plus élevées. Aujourd'hui cette question a été résolue et les hybrides aussi bien ogu-INRA que MSL (autre système d'hybridation développé par la société allemande NPZ-Lembke) ont progressé pour atteindre des niveaux le plus souvent inférieurs à $15 \mu \mathrm{M} / \mathrm{g}$ comme le montre les suivis annuels réalisés sur la collecte par I'ONIDOL et le CETIOM (figure 5).

Le progrès génétique a porté également sur la diversification des profils d'acides gras. Les travaux ont été menés aussi bien à partir de ressources génétiques originales, qu'après mutagénèse ou en utilisant des stratégies OGM. Ces travaux ont abouti commercialement à trois types de profils d'acides gras originaux :

Les variétés riches en acide érucique Si les variétés éruciques ont été exclues du marché de commodité, il reste pour l'industrie un marché érucique souhaitant des variétés à haute teneur en C22-1 et à faible niveau de glucosinolates. Une étape importante a été franchie dans les années 90 avec l'arrivée de la variété "Zéruca» première variété d'hiver érucique et à basse teneur en glucosinolates. Depuis, les rendements ont été

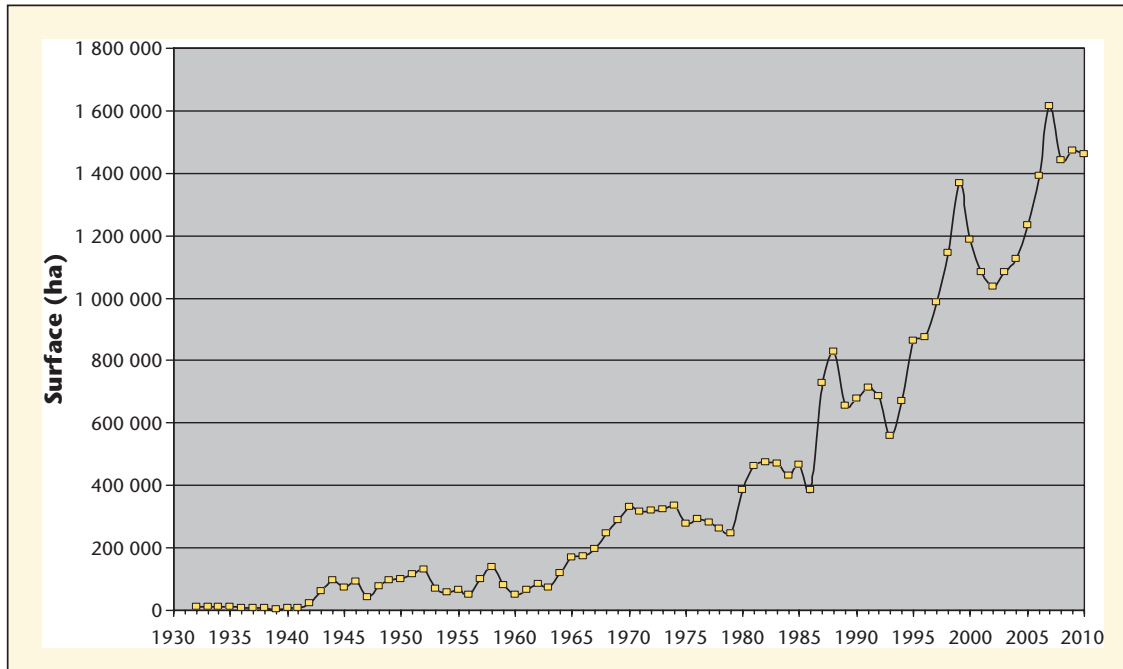

Figure 1. Evolution des surfaces de colza en France de 1932 à 2010. améliorés avec des hybrides (Rossini, Renard) ou avec des lignées portant la résistance spécifique au Phoma RIm7 (Caïman, Heaven). Les teneurs en C22-1 ont également progressées pour franchir beaucoup plus régulièrement la barre de $50 \%$ de C22-1. Par transfert de gènes d'acyltransférase et d'élongase des lignées expérimentales contenant $72 \%$ de C22-1 ont été obtenues.

Les variétés riches en acide laurique

Ces variétés présentent des teneurs en C12-0 (acide laurique) de 30 à $40 \%$. Elles ont été obtenues par transforma- tion par une société de Biotechnologie américaine en introduisant un gène de thioestérase du Laurier de Californie. Des essais mutilocaux ont été conduits à assez large échelle en Europe au milieu des années 90. En France, seuls quelques essais expérimentaux ont été conduits. Depuis face à l'huile de palme cette technologie a été abandonnée faute de compétitivité économique.

Les variétés riches en acide oléique et à faible teneur en acide linolénique Plusieurs variétés à basse teneur en acide linolénique $(\mathrm{C} 18-3<3,5 \%)$ ont été

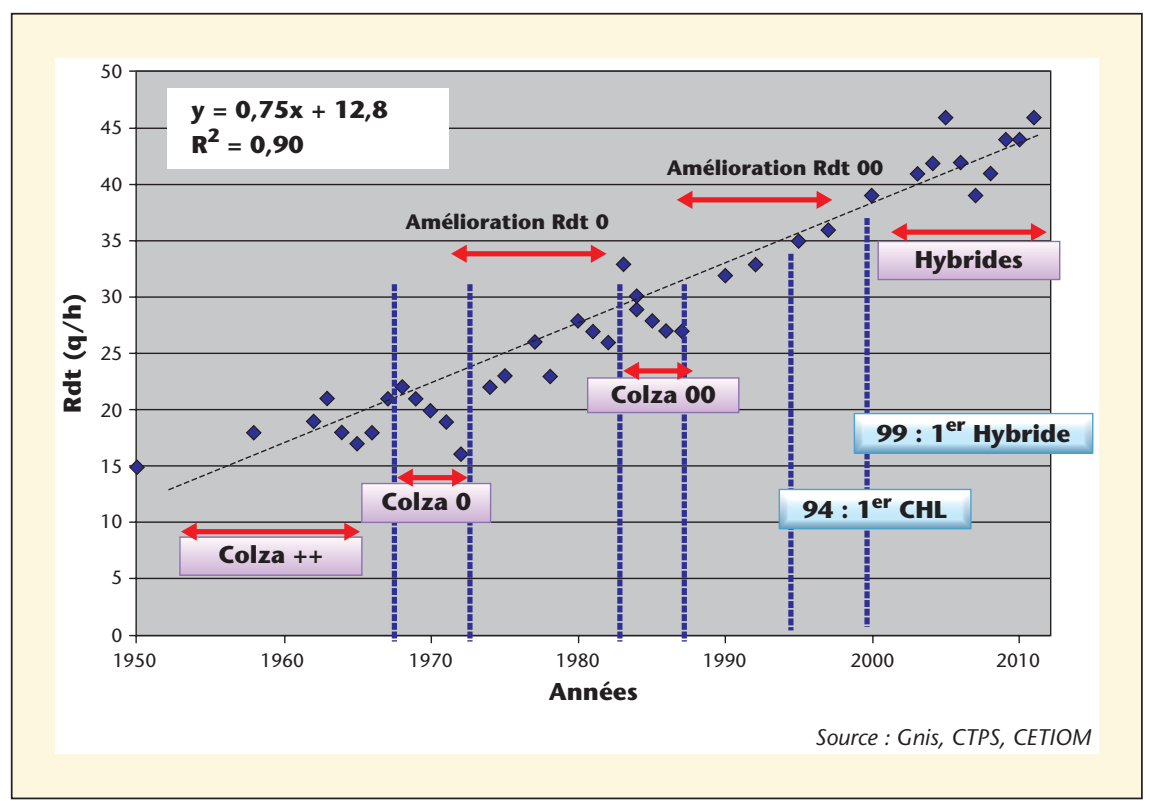

Figure 2. Progrès génétique sur le rendement tel qu'observé sur les réseaux d'inscription du CTPS. 


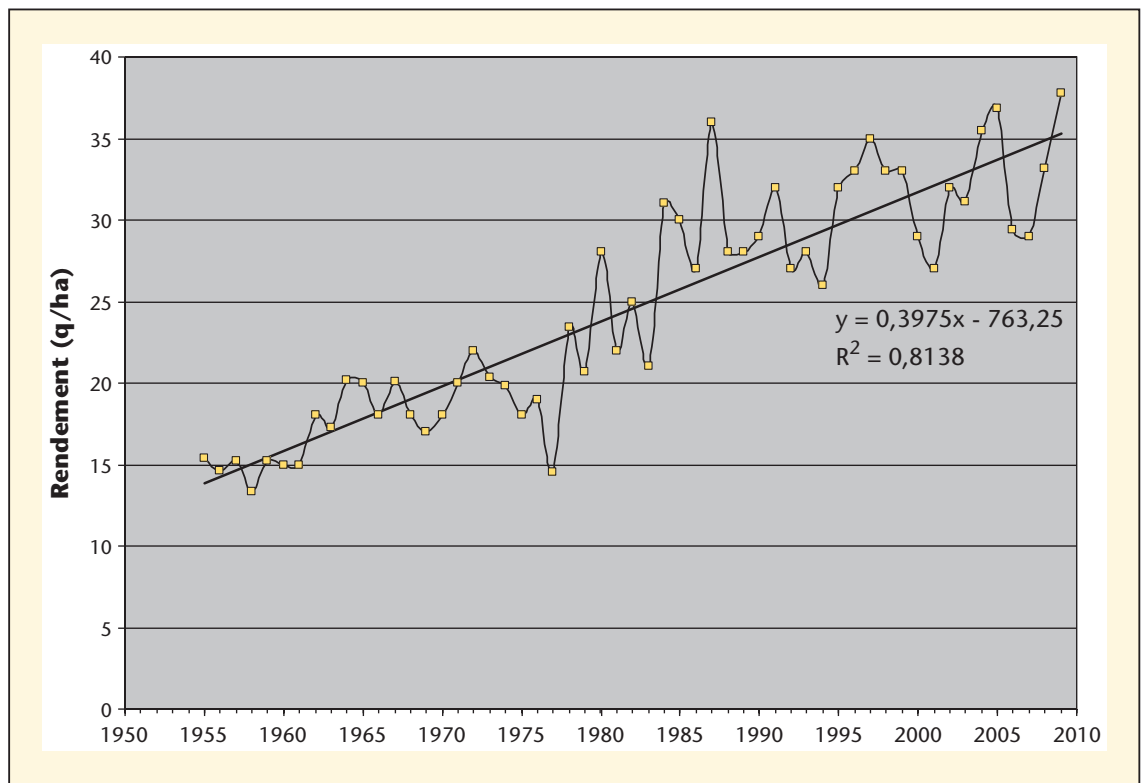

Figure 3. Evolution des rendements moyens nationaux en colza de 1955 à 2009.

inscrites au CTPS au début des années 2000. "Splendor » est la première variété oléique $(\mathrm{C} 18-1>75 \%)$ et bas linolénique $(\mathrm{C} 18-3<3,5 \%)$ inscrite au CTPS. Cette variété cultivée sous contrat, présente un déficit de rendement par rapport aux variétés conventionnelles de l'époque de l'ordre de $15 \%$. Depuis les sociétés de semences impliquées sur ces marchés font des efforts pour améliorer le niveau de productivité. Guguin et al. (2011) ont présenté des résultats suggérant qu'ils

ont créé des hybrides haut oléiques et bas linoléniques présentant des niveaux de rendement équivalents aux hybrides conventionnels et qui devraient donc permettre l'extension des surfaces de ce type de matériel, limitées pour l'instant à quelques milliers d'hectares.

\section{Autres caractères}

D'autres caractères ont fait l'objet d'amélioration. On peut citer la résistance à la verse, d'une façon générale, mais

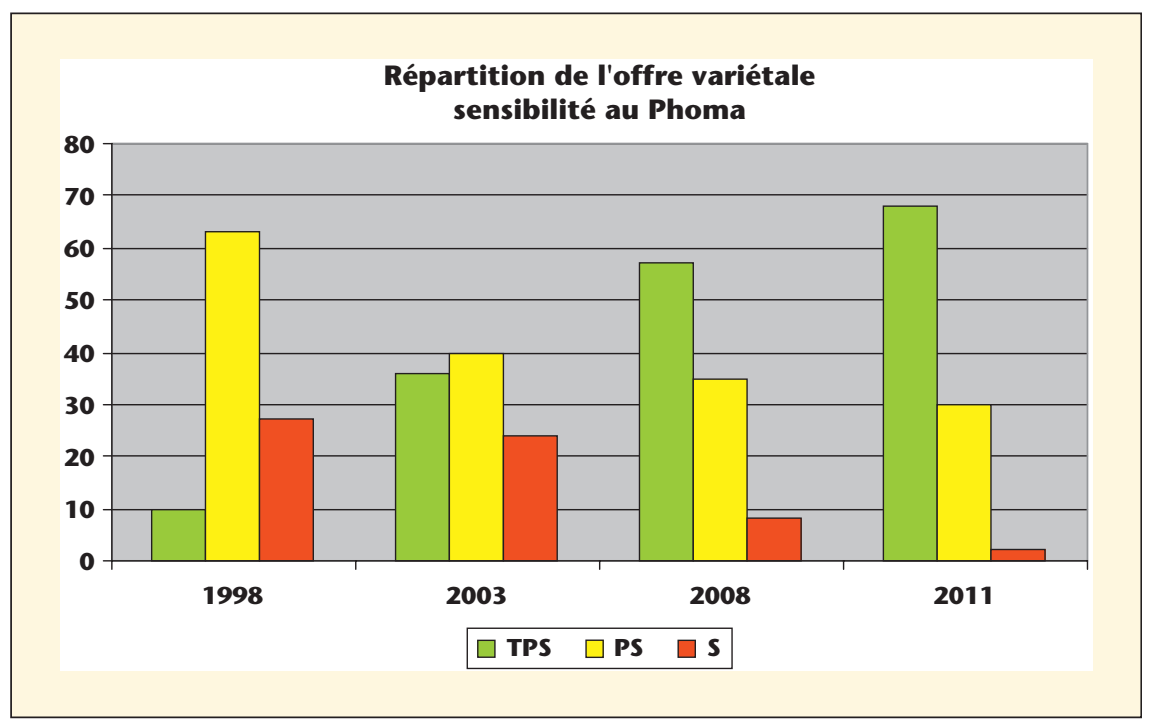

Figure 4. Evolution de la répartition de l'offre variétale en colza entre les 3 classes de sensibilité utilisées, telle que décrite dans les brochures du CETIOM après évaluation des nouvelles variétés au CTPS et au CETIOM. TPS : très peu sensible; PS : peu sensible; $S$ : sensible. aussi à travers l'émergence depuis 1999 d'hybrides demi-nains très résistants. Néanmoins ce type de matériel est peu commercialisé en France alors qu'il a des parts de marché plus importantes dans les pays d'Europe de l'Est où il semble présenter également une meilleure résistance au froid. En France la différence de rendement subsistant avec les dernières générations $d$ 'hybrides conventionnels reste un obstacle à la diffusion, malgré les avantages de résistance à la verse et de gain de vitesse de récolte.

Des lignées à pétales atrophiés obtenues par mutagénèse spontanée ou induite présentent un avantage pour réduire le risque Sclérotinia en réduisant le vecteur de contamination que constitue le pétale.

Par la même technique, ont été générées des lignées cléistogames dont les fleurs restent en forme de tulipe favorisant ainsi l'autofécondation et limitant les émissions de pollen. Ce caractère pourrait avoir un intérêt pour gérer des coexistences entre colza OGM et non OGM ou entre variétés de profils d'acides gras différents.

\section{Et maintenant ?}

Depuis quelques années, la productivité n'est plus le seul objectif. La société est de plus en plus exigeante vis-à-vis de l'agriculture. II faut produire toujours davantage avec des marchés diversifiés s'intéressant à la fois à l'alimentation humaine, I'alimentation animale, et de plus en plus aux applications non alimentaires comme les biocarburants ou la chimie verte en substitution aux produits pétroliers. Ceci doit être fait en respectant des règles d'impacts environnementaux sur les sols, les eaux, les émissions de gaz à effets de serre, en améliorant les bilans énergétiques, et en réduisant I'utilisation de produits chimiques de protection des plantes. Face à ces défis nombreux et difficiles, la génétique apparaît comme un levier de premier plan, si elle arrive à hiérarchiser ses objectifs et répartir ses moyens. Les pouvoirs publics encouragent ces évolutions. Depuis 2010, le CTPS a fait évoluer les épreuves VAT $d$ 'inscription en VATE (valeur agronomique, technologique et environnementale). Le comité plénier du CTPS a demandé à chaque section de décliner en calendriers 


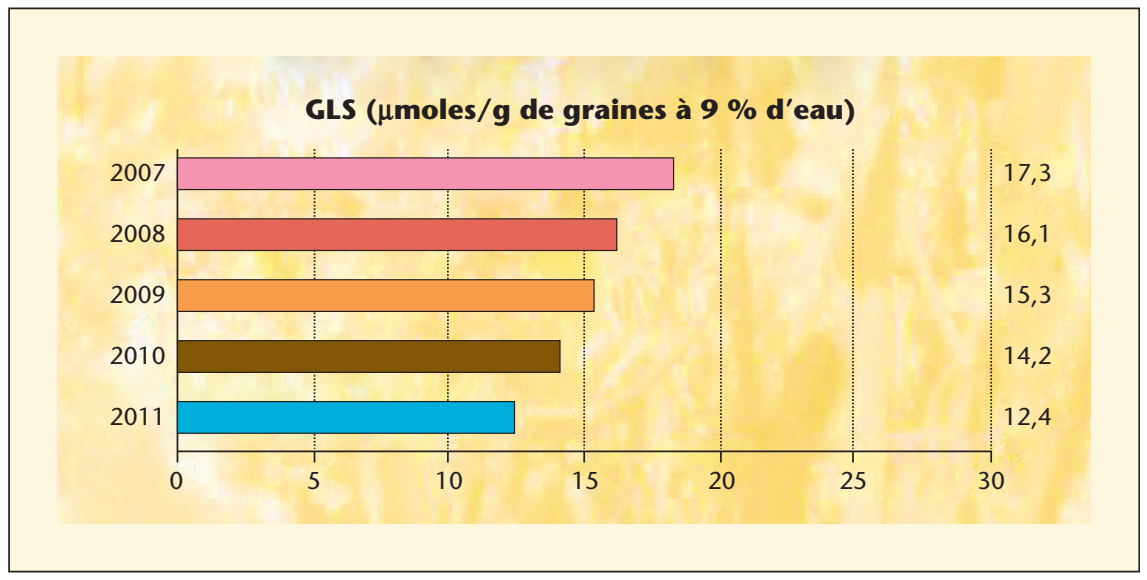

Figure 5. Estimation du niveau de glucosinolates des graines de colza récoltés ces dernières années, sur la base de l'échantillonnage annuel chez les organismes de collecte réalisé par I'ONIDOL et le CETIOM.

d'objectifs pluriannuels cet objectif pour lui donner un contenu concret.

Néanmoins, la productivité reste un objectif majeur pour lequel des approches nouvelles doivent être déployées. Il s'agit principalement des points suivants :

- Optimiser I'hétérosis du matériel hybride (gain par rapport à la moyenne des deux parents) en constituant des populations pour sélectionner séparément les mâles et les femelles et faire en sorte que ces groupes hétérotiques expriment I'hétérosis maximum.

- Élargir la variabilité génétique : Actuellement la base génétique de sélection du colza est considérée comme assez étroite. Un travail d'élargissement des ressources génétiques est donc nécessaire. Outre l'enrichissement avec des sources encore non utilisées de B.napus, ce travail peut aussi s'effectuer en utilisant des ressources génétiques de navette ( $B$.rapa, génome $A$ ) et de chou (B.oleracea, genome C). Ensuite il y a plusieurs possibilités de valorisation de ces ressources en B.napus. Il existe des cas connus de variétés synthétiques construites en réassociant les deux génomes. Néanmoins des études récentes montrent qu'il est plus intéressant de croiser des choux ou des navettes avec un colza pour produire des plantes à 3 génomes AAC ou CCA. Ensuite on peut revenir au colza en sélectionnant, au fil des rétrocroisements, des plantes à $\mathrm{n}=38$. Cette stratégie a l'avantage d'augmenter très sensiblement les recombinaisons lors des méioses et ainsi d'aller plus vite dans l'introgression d'allèles nouveaux.
- Améliorer l'efficience des métabolismes de base de la croissance et du développement d'une plante. II s'agit d'améliorer les 2 fonctions clés liées entre elles que sont, d'une part la nutrition azotée et d'autre part l'interception du rayonnement lumineux et l'activité photosynthétique pour la nutrition en carbone. Cela se joue en partie par des aspects architecturaux des plantes pour optimiser cette interception du rayonnement.

- Réduire l'égrainage. Cette idée part du constat de pertes à la récolte importantes et mal maîtrisées. Les tentatives de chiffrage de ces pertes les évaluent de 2 à 5 quintaux et facilement beaucoup plus dès qu'il y a une difficulté. Une partie de ces pertes peut être évitée par des aménagements de tablier de moissonneuse batteuse. Néanmoins une fraction de 20 à $30 \%$ des pertes estimées échappe à la récolte. D'un point de vue physiologique, les mécanismes de déhiscence de la silique sont connus et certains allèles correspondants sont identifiés. La difficulté réside dans la détermination du bon équilibre entre déhiscence prématurée et mauvais battage, les deux conduisant à des pertes de récolte. Certains hybrides produits avec le système OguINRA présenteraient une plus grande résistance à l'égrainage.

La génétique a aussi un rôle primordial à jouer pour les objectifs du plan ECOPHYTO 2018. Les objectifs de recherche de résistances aux bioagresseurs s'en trouvent naturellement renforcés. Audelà, la réduction des intrants (azote, produits phytosanitaires, régulateurs de croissance, etc.) amène logiquement une hypothèse d'interactions génotypes $\times$ milieu $x$ conduites plus importantes aussi bien en ampleur qu'en fréquence. Aujourd'hui, les approches de modélisation facilitent l'étude de ces interactions et leur maitrise de façon à utiliser la bonne variété au bon endroit et avec le bon mode d'emploi. Les stratégies de protection ne peuvent pas non plus rester cantonnées à l'échelle de la parcelle, mais doivent envisager des échelles plus larges à la fois à l'échelle des paysages et dans le temps. Ces stratégies de protection peuvent faire appel et utiliser des caractères variétaux divers allant bien au-delà des seules résistances aux maladies ou aux insectes, mais peuvent s'intéresser à une variabilité disponible sur des caractères comme la vigueur au départ, la précocité, la durée entre stades de développement, la hauteur, des caractères morphologiques, I'émission de substances volatiles issus de métabolismes secondaires.

Pour le colza reste deux thématiques primordiales qui vont être largement abordées dans les années qui viennent.

La première est le contrôle des insectes : Charançons, Altises, Pucerons, méligèthes. L'IFT moyen en colza (Indice de fréquence de traitement) est voisin de 6 dont 3 pour les seuls insecticides. II est donc clair que la diminution de ces applications insecticides est une priorité. Les stratégies à développer nécessitent des acquisitions de connaissances complémentaires sur l'écologie des différentes populations d'insectes non seulement dans la parcelle, mais aussi en dehors. Ces stratégies seront très probablement conçues à l'échelle du paysage en tenant compte des zones non cultivées, des pratiques et des dynamiques d'insectes parasitoïdes associés. Néanmoins elles feront également nécessairement appel à des caractères des variétés comme une variabilité sur les dates de floraison, ou sur l'émission de métabolites secondaires pour développer des stratégies de type Push and Pull.

Le second sujet majeur est l'optimisation de l'efficience de la nutrition azotée. Cette thématique est à la fois commanditée par des considérations d'impacts environnementaux, économiques, et d'amélioration des bilans énergétiques des filières non alimentaires utilisant I'huile de colza. En particulier la directive 
européenne sur l'énergie fixe des contraintes importantes qui motivent des projets non seulement en France, mais aussi en Angleterre et en Allemagne. Cette thématique fait aujourd'hui l'objet d'un gros projet (2012-2019) rassemblant la plupart des équipes publiques et privés travaillant sur ce sujet au niveau national. II vient d'être accepté pour financement dans le cadre des appels à projet investissements $d$ 'avenir financés par le grand emprunt.

\section{RÉFÉRENCES}

Agence canadienne d'inspection des aliments. DD1995-04 évaluation du risque environnemental associé à une nouvelle méthode d'hybridation du colza (B.napus L.) de Plant Genetic Systems Inc. (PGS). www.inspection. gc.ca.

Ahman I, Lehrman A, Ekbom B. Impact of herbivory and pollination on performance and competitive ability of oilseed rape transformed for pollen beetle resistance. Arthropod-Plant Interactions 2009 ; 3 : 105-13.

Cartea ME, Migdal M, Galle AM, Pelletier G, Guerche P. Comparison of sense and antisense methodologies for modifying the fatty acid composition of Arabidopsis thaliana oilseed. Plant Science $1998 ; 136$ : 18194.

Dong X, Ji R, Guo X, et al. Expressing a gene encoding wheat oxalate oxidase enhances resistance to Sclerotinia sclerotiorum in oilseed rape (Brassica napus). Planta 2008 ; 228 : 331 40.

Dröge W, Broerl, Pühler A. Transgenic plants containing the phosphinothricin- $\mathrm{N}$-acetyltransferase gene metabolize the herbicide L-Phosphinothricin (glufosinate) differently from untransformed plants. Planta 1992 ; 187 : 142-51.

Freyssinet G, Pelissier B, Freyssinet M, Delon R. Crops resistant to oxynils: from the laboratory to the market. Field Crops Research $1996 ; 45: 125-33$.
Freyssinet M, Dumas B, Sailland A, Pepin R, Freyssinet $G$. Transgenic crops expressing oxalate oxidase as a way to incease resistance to oxalate producing pathogens. 9th International Rape Seed Congress Cambridge $1995 ; 4: 1278-80$.

Grezes-Besset B, Grison R, Villeger MJ, Nicolas $C$, Toppan A. Field testing against four fungal pathogens of transgenic Brassica napus plants constitutively expressing a chitinase gene. 9th International Rape Seed Congress Cambridge 1995 ; 3 : 781-3.

Grison R, Grezes-Besset B, Schneider M, Toppan A. Field tolerance to fungal pathogens of Brassica napus constitutively expressing a chimeric chitinase gene. Nature Biotechnology 1996 ; 14 : 643-46.

Good AG, Johnson SJ, De Pauw M, et al. Engineering nitrogen use efficiency with alanine aminotransferase. Can J Bot 2007 ; $85: 252-62$.

Guguin N, Lehmann L, Richter A, Busch H, Despeghel JP. Breeding and development of HOLL winter oilseed rape hybrids. $13^{\text {th }}$ international Rapeseed Congress Prague June 5-9 2011: 566-8.

Hua S, Shamsi IH, Guo Y, et al. Sequence expression divergence, and complementation of homologous ALCATRAZ loci in Brassica napus. Planta 2009 ; 230 : 493-503.

Jouanin L, Bonadé-Bottino M, Girard C, Morrot G, Giband M. Transgenic plants for insect resistance. Plant Sci 1998 ; 131 : 1-11.

Luciani $A$. Etude du progrès génétique chez différentes espèces de grandes cultures. GEVES : Ed La Minière, 2004.

Nath UK, Wilmer JA, Wallington EJ, Becker HC, Möllers C. Increasing erucic acid content through combination of endogenous low polyunsaturated fatty acids alleles with LdLPAAT + Bn-fae1 transgenes in rapeseed (Brassica napus L.). TAG 2009 ; 118 : 765-73.

Ostergaard L, Kempin S, Bies D, Klee H, Yanofsky M. Pod shattering-resistant Brassica fruit produced by ectopic expression of the FRUITFALL gene. Plant Biotechnol / 2006 ; 4 : 45-51.
Pelletier G, Primard C, Vedei Chetrit P, Remy R, Rousselle P, Renard M. Intergeneric cytoplasmic hybridization in Cruciferae by protoplast fusion. Molecular and General Genetics 1983 ; 191 : 244-50.

Reiss E, Schubert J, Scholze P, Kramer R, Sonntag K. The barley thaumatin-like protein Hv-TLP8 enhances resistance of oilseed rape plants to Plasmodiophora brassicae. Plant Breeding $2009 ; 128$ : 210-2.

Rudloff et Wehling, 1997 Release of transgenic oilseed rape (Brassica napus L.) with altered fatty acids ISHS Acta Horticulturae 459 : International Symposium Brassica 97, Xth Crucifer Genetics Workshop.

Shah DM, Rogers SG, Horsch RB, Fraley RT Glyphosate resistant plant. US Patent $n^{\circ} 4$ 940 835. July 1990.

Simard MJ, Légère A, Pageau D, Lajeunesse J, Warwick SI. The frequency and persistence of volunteer canola (Brassica napus) in Quebec cropping systems. Weed Technol 2002; 16 : 433-9.

Stewart CN Jr, Adang MJ, Ail JN, Raymer PL, Ramachandran S, Parrott WA. Insect control and dosage effects in transgenic canola, Brassica napus L. (Brassicaceae), containing a synthetic Bacillus thuringiensis crylA(c) gène. Plant Physiology 1996 ; 112 : 115-20.

Thompson C, Dunwell JM, Johnstone CE, et al. Degradation of oxalic acid by transgenic oilseed rape plants expressing oxalate oxidase. Euphytica 1995 ; 85 : 169-72.

Voelker TA, Worrell AC, Anderson L, et al. Fatty acid biosynthesis redirected to medium chains in transgenic oilseed plants. Science 1992 ; 257 : 72-4.

Wang Y, Fristensky B. Transgenic canola lines expressing pea defense gene DRR206 have resistance to aggressive blackleg isolates and to Rhizoctonia solani. Molecular Breeding $2001 ; 8: 263-71$.

Yang $X$, Wang $H$, Lui G, Wang $X$. The lignin biosynthesis regulation and its relationship with Sclerotinia and lodging resistances for Brassica napus. 12th International Rape Seed Congress Wuhan 2007 ; 2 : 50-2. 